\title{
Surface wave propagation in a fluid saturated incompressible porous half-space lying under two layers of different liquids
}

\author{
Rajneesh Kumar $^{1}$ and B. S. Hundal ${ }^{2}$ \\ ${ }^{1}$ Department of Mathematics, Kurukeshetra University, Kurukeshetra, Haryana, India \\ ${ }^{2}$ Department of Mathematics, S. R. Government College for Women, Amritsar, Punjab, India \\ (Received September 28, 2006; Revised May 14, 2007; Accepted June 28, 2007; Online published August 31, 2007)
}

\begin{abstract}
A study of surface wave propagation in a fluid saturated incompressible porous half-space lying under a double-layer consisting of non-homogeneous and homogeneous liquids is presented. The frequency equation connecting the phase velocity with wave number is derived. Special cases as: (i) Rayleigh type surface waves in an incompressible poro-elastic half-space lying under a uniform layer of a homogeneous liquid, (ii) Rayleigh type surface waves in an incompressible poro-elastic half-space lying under a uniform layer of a non-homogeneous liquid and (iii) Rayleigh type surface waves propagating along the free surface of a fluid saturated incompressible porous elastic half-space, are investigated. Numerical results with graphical presentations of the variations of phase velocity with wave number for different cases are also included.
\end{abstract}

Key words: Incompressible porous medium, volume fractions, frequency equation, phase velocity, wave number.

\section{Introduction}

From the geophysical and acoustic point of view, the propagation of elastic waves in a layered half-space is of considerable interest. In seismology especially in seismic wave propagation there are many cases when the medium is composed of two or more layers lying over elastic halfspace and generally this half-space is not a single-phase model. Beside this most of the other modern engineering structures are generally made up of multiphase porous continuum and the classical theory, which represents a fluid saturated porous medium as a single phase material, is inadequate to represent the mechanical behavior of such materials especially when the pores are filled with liquid. In this case the solid and liquid phases have the different motions. Due to these different motions, the different material properties and the complicated geometry of pore structure the mechanical behavior of a fluid saturated porous medium becomes more difficult. So from time to time, researchers have tried to overcome this difficulty and considerable work has been done in this regard. For more detail and for the historical review on the subject of multiphase continuum mechanics, the reader is referred to the work of Boer and Ehlers (1988) and Boer (1996, 2000a, b).

Based on the work of von Terzaghi (1923, 1925), Biot (1941) proposed a general theory of three-dimensional deformations of fluid saturated porous elastic solids. Then in the subsequent work, Biot (1956a, b, 1962) did the dynamic extension of his theory and proposed the propagation of two dilatational and one rotational elastic waves in the fluid saturated porous solids. Biot theory is based on the assumption of compressible constituents and till recently, some of

Copyright (c) The Society of Geomagnetism and Earth, Planetary and Space Sciences (SGEPSS); The Seismological Society of Japan; The Volcanological Society of Japan; The Geodetic Society of Japan; The Japanese Society for Planetary Sciences; TERRAPUB his results have been taken as standard references and the basis for subsequent analysis in acoustic, geophysics and other such fields.

Based on the Fillunger model (1933), (which is further based on the concept of volume fractions combined with surface porosity coefficients), Bowen (1980), Boer and Ehlers (1990a, b) and Ehlers (1993) developed and used another interesting theory in which all the constituents of a porous medium are assumed to be incompressible. There are reasonable grounds for the assumption that the constituents of many fluid saturated porous media are incompressible. For example, taking the composition of soil; solid constituents are incompressible and liquid constituents which are generally water or oils are also incompressible. Moreover in an empty porous solid as a case of classical theory, the change in volume is due to the change in porosity during the propagation of a longitudinal wave. The assumption of incompressible constituents does not only meet the properties appearing in many branches of engineering practice, but it also avoids the introduction of many complicated material parameters as considered in the Biot theory. So this type of models, meet the assumptions and requirements of further scientific developments. Based on this theory Boer et al. (1993), Boer and Liu (1996), Liu and Boer (1994, 1995, 1997), Liu (1999), Yan et al. (1999), and Kumar and Hundal (2002, 2003a, b, 2004a, b) have studied the problems of wave propagation in fluid saturated incompressible porous media.

Many investigators have discussed the surface wave propagation in elastic media and a comprehensive review is available in the standard texts, e.g. Ewing et al. (1957) and Achenbach (1976) etc. The surface waves discussed in these texts are within the scope of single-phase models, but the presence of fluid in the pores of an elastic porous solid might have affected the motion of solid particles. As 
for as the multi-phase systems are concerned; there is considerable work concerning the surface wave propagation in fluid-saturated porous media at the present time, and a brief review is available in the work of Kumar and Miglani (1996), Kumar et al. (2002), Liu and Liu (2004) and Edelman (2004). But all this work is based on the classical Biot model where the constituents of a fluid-saturated porous medium are assumed to be compressible. However, Liu and Boer (1997) have discussed the wave propagation characteristics including dispersion and attenuation of Rayleigh and Love type waves in a fluid saturated incompressible porous medium.

Oceanic models involving double liquid layer of inhomogeneous and homogeneous liquids lying over the fluid saturated soil and other materials, which are porous but incompressible in nature, are often present in the Earth model. So in the present study we have tried to investigate the surface wave propagation in one such model by taking first layer to be non-homogeneous, with non-homogeneity varying with depth, and the second layer homogeneous. The half-space is taken as a two-phase system with incompressible solid phase and an incompressible fluid phase. Frequency equation relating the phase velocity with the wave number and other material parameters is derived and the variations of phase velocity with wave number for different values of the non-homogeneity parameter and for different values of the ratio of the thicknesses of layers are presented graphically and are discussed. Depending upon the thicknesses of the layers, some particular cases have also been included. Results at various steps are compared with classical theories.

\section{Basic Equations}

Within the framework of modern porous media theories (Boer and Ehlers, 1990), the equations governing the deformation of a fluid saturated incompressible porous medium are

$$
\begin{gathered}
\operatorname{div} \cdot\left(\eta^{\mathrm{S}} \dot{\mathbf{x}}_{\mathrm{S}}+\eta^{\mathrm{F}} \dot{\mathbf{x}}_{\mathrm{F}}\right)=\mathbf{0}, \\
\operatorname{div} \cdot \mathbf{T}_{\mathrm{E}}^{\mathrm{S}}-\eta^{\mathrm{S}} \operatorname{grad} \cdot p+\rho^{\mathrm{S}}\left(\mathbf{b}-\ddot{\mathbf{x}}_{\mathrm{S}}\right)-\mathbf{P}_{\mathrm{E}}^{\mathrm{F}}=\mathbf{0}, \\
\operatorname{div} \cdot \mathbf{T}_{\mathrm{E}}^{\mathrm{F}}-\eta^{\mathrm{F}} \operatorname{grad} . p+\rho^{\mathrm{F}}\left(\mathbf{b}-\ddot{\mathbf{x}}_{\mathrm{F}}\right)+\mathbf{P}_{\mathrm{E}}^{\mathrm{F}}=\mathbf{0},
\end{gathered}
$$

where $\dot{\mathbf{x}}_{i}$ and $\ddot{\mathbf{x}}_{i}(i=\mathrm{F}, \mathrm{S})$ denote the velocities and accelerations of solid and fluid phases respectively and $p$ is the effective pore pressure of the incompressible pore fluid. $\rho^{\mathrm{S}}$ and $\rho^{\mathrm{F}}$ are the densities of the solid and fluid phases respectively and $\mathbf{b}$ is the body force per unit volume. $\mathbf{T}_{\mathrm{E}}^{\mathrm{S}}, \mathbf{T}_{\mathrm{E}}^{\mathrm{F}}$ and $\mathbf{P}_{\mathrm{E}}^{\mathrm{F}}$ are called the extra quantities for which the constitutive equations must be formulated and $\eta^{\mathrm{S}}, \eta^{\mathrm{F}}$ are the volume fractions satisfying

$$
\eta^{\mathrm{S}}+\eta^{\mathrm{F}}=1
$$

If $\mathbf{u}_{\mathrm{S}}$ and $\mathbf{u}_{\mathrm{F}}$ are the displacement vectors for solid and fluid phases then

$$
\dot{\mathbf{x}}_{\mathrm{S}}=\dot{\mathbf{u}}_{\mathrm{S}}, \ddot{\mathbf{x}}_{\mathrm{S}}=\ddot{\mathbf{u}}_{\mathrm{S}}, \quad \dot{\mathbf{x}}_{\mathrm{F}}=\dot{\mathbf{u}}_{\mathrm{F}}, \ddot{\mathbf{x}}_{\mathrm{F}}=\ddot{\mathbf{u}}_{\mathrm{F}}
$$

The investigations to follow are restricted to a linear isotropic, elastic incompressible porous medium filled with an inviscid liquid. So the constitutive equations for extra stresses and extra momentum are given by Boer and Ehlers (1990) as

$$
\begin{aligned}
& \mathbf{T}_{\mathrm{E}}^{\mathrm{S}}=2 \mu^{\mathrm{S}} \mathbf{E}_{\mathrm{S}}+\lambda^{\mathrm{S}}\left(\mathbf{E}_{\mathrm{S}} . \mathbf{I}\right) \mathbf{I}, \\
& \mathbf{T}_{\mathrm{E}}^{\mathrm{F}}=\mathbf{0}, \\
& \mathbf{P}_{\mathrm{E}}^{\mathrm{F}}=-\mathbf{S}_{\mathrm{V}}\left(\dot{\mathbf{u}}_{\mathrm{F}}-\dot{\mathbf{u}}_{\mathrm{S}}\right),
\end{aligned}
$$

where $\lambda^{\mathrm{S}}$ and $\mu^{\mathrm{S}}$ are the macroscopic Lame's parameters of the porous solid and $\mathbf{E}_{\mathrm{S}}$ is the linearized Langrangian strain tensor defined as

$$
\mathbf{E}_{\mathrm{S}}=\frac{1}{2}\left(\operatorname{grad} \mathbf{u}_{\mathrm{S}}+\operatorname{grad}^{T} \mathbf{u}_{\mathrm{S}}\right)
$$

In the case of isotropic permeability, the tensor $\mathbf{S}_{\mathrm{V}}$ describing the coupled interaction between the solid and fluid is given by Boer and Ehlers (1990) and Boer et al. (1993) as

$$
\mathbf{S}_{\mathrm{V}}=\frac{\left(\eta^{\mathrm{F}}\right)^{2} \gamma^{\mathrm{FR}}}{K^{\mathrm{F}}} \mathbf{I},
$$

where $\gamma^{\mathrm{FR}}$ is the effective specific weight of the fluid and $K^{\mathrm{F}}$ is the Darcy's permeability coefficient of the porous medium.

\section{Formulation of the Problem}

We consider a model consisting of a double layer of two different liquids, resting on a half-space of a fluid saturated incompressible porous medium of infinite extent. The upper layer $\mathrm{L}_{1}$ is non-homogeneous and is of thickness $h$, whereas the lower layer $L_{2}$ is homogeneous and its thickness is taken to be $H$. The coordinate system is selected with $x, y$-plane coinciding with the interface between two layers, $x$-axis along the length and $z$-axis perpendicular to the interface along the direction of increasing depth. So the layers $L_{1}$, $\mathrm{L}_{2}$ and the half-space occupies the regions $-h<z \leq 0$, $0 \leq z<H$ and $z \geq H$ respectively as shown in Fig. 1 . We also consider the waves of plane strain with wave front parallel to the $y$-axis so that the field components in the $y$ direction vanish and are independent of $y$ coordinate.

Also in the scope of infinitesimal deformations, all the terms of higher orders are neglected. Moreover, the small variation in volume fractions is also neglected and for the two dimensional problem we take

$$
\left.\begin{array}{l}
\mathbf{u}^{\mathrm{S}}=\left(u^{\mathrm{S}}, 0, w^{\mathrm{S}}\right) \\
\mathbf{u}^{\mathrm{F}}=\left(u^{\mathrm{F}}, 0, w^{\mathrm{F}}\right)
\end{array}\right\} .
$$

Using Eq. (11), Eqs. (1)-(8) for the half-space in the absence of body forces take the form

$$
\begin{aligned}
\left(\lambda^{\mathrm{S}}+\mu^{\mathrm{S}}\right) \frac{\partial \theta^{\mathrm{S}}}{\partial x}+ & \mu^{\mathrm{S}} \nabla^{2} u^{\mathrm{S}}-\eta^{\mathrm{S}} \frac{\partial p}{\partial x}-\rho^{\mathrm{S}} \frac{\partial^{2} u^{\mathrm{S}}}{\partial t^{2}} \\
& +S_{\mathrm{V}}\left(\frac{\partial u^{\mathrm{F}}}{\partial t}-\frac{\partial u^{\mathrm{S}}}{\partial t}\right)=0 \\
\left(\lambda^{\mathrm{S}}+\mu^{\mathrm{S}}\right) \frac{\partial \theta^{\mathrm{S}}}{\partial z}+ & \mu^{\mathrm{S}} \nabla^{2} w^{\mathrm{S}}-\eta^{\mathrm{S}} \frac{\partial p}{\partial z}-\rho^{\mathrm{S}} \frac{\partial^{2} w^{\mathrm{S}}}{\partial t^{2}} \\
& +S_{\mathrm{V}}\left(\frac{\partial w^{\mathrm{F}}}{\partial t}-\frac{\partial w^{\mathrm{S}}}{\partial t}\right)=0
\end{aligned}
$$




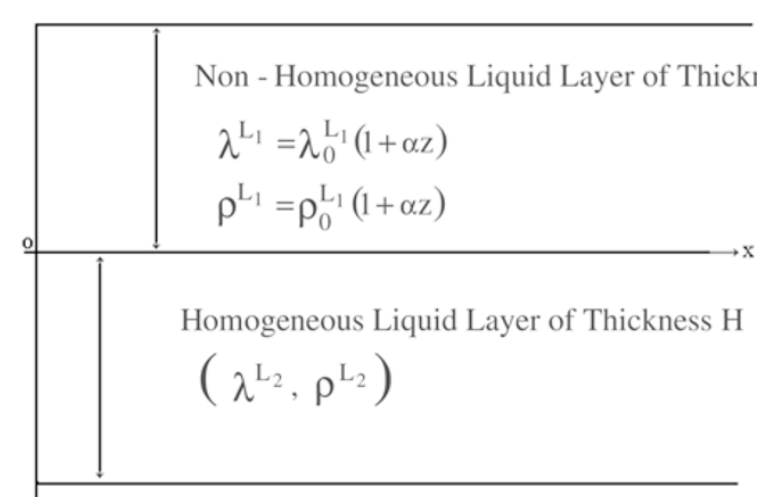

Fluid Saturated Incompressible Porous Half-Space $\left(\lambda^{\mathrm{S}}, \mu^{\mathrm{S}}, \rho^{\mathrm{s}}, \rho^{\mathrm{F}}, \eta^{\mathrm{s}}, \eta^{\mathrm{F}}, \gamma^{\mathrm{FR}}, \mathrm{K}^{\mathrm{F}}\right)$

Fig. 1. Geometry of the investigated problem.

$$
\begin{aligned}
& \eta^{\mathrm{F}} \frac{\partial p}{\partial x}+\rho^{\mathrm{F}} \frac{\partial^{2} u^{\mathrm{F}}}{\partial t^{2}}+S_{\mathrm{V}}\left(\frac{\partial u^{\mathrm{F}}}{\partial t}-\frac{\partial u^{\mathrm{S}}}{\partial t}\right)=0 \\
& \eta^{\mathrm{F}} \frac{\partial p}{\partial z}+\rho^{\mathrm{F}} \frac{\partial^{2} w^{\mathrm{F}}}{\partial t^{2}}+S_{\mathrm{V}}\left(\frac{\partial w^{\mathrm{F}}}{\partial t}-\frac{\partial w^{\mathrm{S}}}{\partial t}\right)=0 \\
& \eta^{\mathrm{S}}\left(\frac{\partial^{2} u^{\mathrm{S}}}{\partial x \partial t}+\frac{\partial^{2} w^{\mathrm{S}}}{\partial z \partial t}\right)+\eta^{\mathrm{F}}\left(\frac{\partial^{2} u^{\mathrm{F}}}{\partial x \partial t}+\frac{\partial^{2} w^{\mathrm{F}}}{\partial z \partial t}\right)=0
\end{aligned}
$$

where

$$
\theta^{\mathrm{S}}=\frac{\partial u^{\mathrm{S}}}{\partial x}+\frac{\partial w^{\mathrm{S}}}{\partial z} .
$$

For the layers $\mathrm{L}_{1}$ and $\mathrm{L}_{2}$, equations governing the motions of liquids are given by Ewing et al. (1957) as

$$
\begin{gathered}
\lambda^{\mathrm{L}_{i}} \nabla\left(\nabla \mathbf{u}^{\mathrm{L}_{i}}\right)=\rho^{\mathrm{L}_{i}} \frac{\partial^{2} \mathbf{u}^{\mathrm{L}_{i}}}{\partial t^{2}}, \\
\tau_{m n}^{\mathrm{L}_{i}}=\lambda^{\mathrm{L}_{i}} \nabla u^{\mathrm{L}_{i}} \delta_{m n},
\end{gathered}
$$

where $m, n=1,2,3$ and $i=1,2$ refer to layers $\mathrm{L}_{1}$ and $\mathrm{L}_{2}$ respectively. In these two equations $\mathbf{u}^{\mathrm{L}_{i}}$ are the displacement vectors, $\lambda^{\mathrm{L}_{i}}$ are the bulk modulii of the liquids, $\rho^{\mathrm{L}_{i}}$ are their densities and $\tau_{m n}^{\mathrm{L}_{i}}$ are the components of the stresses in the liquids. For the present problem, the displacement vectors $\mathbf{u}^{\mathrm{L}_{i}}=\left(u^{\mathrm{L}_{i}}, 0, w^{\mathrm{L}_{i}}\right)$. So Eqs. (18) and (19) for the layers $\mathrm{L}_{1}$ and $\mathrm{L}_{2}$ are simplified as:

$$
\begin{aligned}
& \frac{\partial}{\partial x}\left(\lambda^{\mathrm{L}_{1}} \theta^{\mathrm{L}_{1}}\right)=\rho^{\mathrm{L}_{1}} \frac{\partial^{2} u^{\mathrm{L}_{1}}}{\partial t^{2}}, \\
& \frac{\partial}{\partial z}\left(\lambda^{\mathrm{L}_{1}} \theta^{\mathrm{L}_{1}}\right)=\rho^{\mathrm{L}_{1}} \frac{\partial^{2} w^{\mathrm{L}_{1}}}{\partial t^{2}}, \\
& \tau_{z z}^{\mathrm{L}_{1}}=\tau_{x x}^{\mathrm{L}_{1}}=\lambda^{\mathrm{L}_{1}} \theta^{\mathrm{L}_{1}},
\end{aligned}
$$

$$
\begin{aligned}
& \lambda^{\mathrm{L}_{2}} \frac{\partial \theta^{\mathrm{L}_{2}}}{\partial x}=\rho^{\mathrm{L}_{2}} \frac{\partial^{2} u^{\mathrm{L}_{2}}}{\partial t^{2}}, \\
& \lambda^{\mathrm{L}_{2}} \frac{\partial \theta^{\mathrm{L}_{2}}}{\partial z}=\rho^{\mathrm{L}_{2}} \frac{\partial^{2} w^{\mathrm{L}_{2}}}{\partial t^{2}}, \\
& \tau_{z z}^{\mathrm{L}_{2}}=\tau_{x x}^{\mathrm{L}_{2}}=\lambda^{\mathrm{L}_{2}} \theta^{\mathrm{L}_{2}},
\end{aligned}
$$

where

$$
\begin{aligned}
& \theta^{\mathrm{L}_{1}}=\frac{\partial u^{\mathrm{L}_{1}}}{\partial x}+\frac{\partial w^{\mathrm{L}_{1}}}{\partial z}, \\
& \theta^{\mathrm{L}_{2}}=\frac{\partial u^{\mathrm{L}_{2}}}{\partial x}+\frac{\partial w^{\mathrm{L}_{2}}}{\partial z} .
\end{aligned}
$$

Beside this, let the non-homogeneity of the layer $\mathrm{L}_{1}$ be defined as

$$
\lambda^{\mathrm{L}_{1}}=\lambda^{\mathrm{L}_{0}^{1}}(1+\alpha z), \quad \rho^{\mathrm{L}_{1}}=\rho^{\mathrm{L}_{0}^{1}}(1+\alpha z) .
$$

\section{Boundary Conditions}

The boundary conditions for the present problem are as follows:

(a) Boundary of the upper layer is stress free so

$$
\tau_{z z}^{\mathrm{L}_{1}}=0 \quad \text { at } z=-h
$$

(b) Conditions at the interface between two layers are the continuity of normal stresses and the displacement components. So at the interface $z=0$, we have

$$
\left.\begin{array}{l}
\text { (i) } \tau_{z z}^{\mathrm{L}_{1}}=\tau_{z z}^{\mathrm{L}_{2}} \\
\text { (ii) } w^{\mathrm{L}_{1}}=w^{\mathrm{L}_{2}}
\end{array}\right\} \quad \text { at } z=0
$$

(c) At the interface $z=H$, continuity of normal stresses, vanishing of shear stress in the saturated incompressible poro-elastic half-space and the continuity of displacements provide

$$
\left.\begin{array}{ll}
\text { (i) } & \tau_{z z}^{\mathrm{L}_{2}}=\tau_{z z}^{\mathrm{S}}-p \\
\text { (ii) } & \tau_{x z}^{\mathrm{S}}=0 \\
\text { (iii) } & w^{\mathrm{L}_{2}}=w^{\mathrm{S}}
\end{array}\right\} \quad \text { at } z=H \text {. }
$$

\section{Derivation of Frequency Equation}

The equations (12)-(16), (20)-(28) along with the conditions (29)-(31) govern the propagation of surface waves in the model consisting of an incompressible porous medium half-space, lying under a double-layer of non-homogeneous and homogeneous liquids. For further considerations, it is convenient to introduce in Eqs. (12)-(31), the dimensionless quantities defined as:

$$
\begin{aligned}
& x^{\prime}=\frac{\omega^{\prime}}{c_{1}} x, \quad z^{\prime}=\frac{\omega^{\prime}}{c_{1}} z, \quad t^{\prime}=\omega^{\prime} t, \\
& u^{\prime \mathrm{S}}=\left(\frac{\lambda^{\mathrm{S}}+2 \mu^{\mathrm{S}}}{E}\right) \frac{\omega^{\prime}}{c_{1}} u^{\mathrm{S}}, w^{\prime \mathrm{S}}=\left(\frac{\lambda^{\mathrm{S}}+2 \mu^{\mathrm{S}}}{E}\right) \frac{\omega^{\prime}}{c_{1}} w^{\mathrm{S}}, \\
& u^{\prime \mathrm{F}}=\left(\frac{\lambda^{\mathrm{S}}+2 \mu^{\mathrm{S}}}{E}\right) \frac{\omega^{\prime}}{c_{1}} u^{\mathrm{F}}, w^{\prime \mathrm{F}}=\left(\frac{\lambda^{\mathrm{S}}+2 \mu^{\mathrm{S}}}{E}\right) \frac{\omega^{\prime}}{c_{1}} w^{\mathrm{F}}, \\
& p^{\prime}=\frac{p}{E}, \quad \tau_{x z}^{\prime}=\frac{\tau_{x z}}{E}, \quad \tau_{z z}^{\prime}=\frac{\tau_{z z}}{E}, \\
& u^{\prime \mathrm{L}_{1}}=\left(\frac{\lambda^{\mathrm{S}}+2 \mu^{\mathrm{S}}}{E}\right) \frac{\omega^{\prime}}{c_{1}} u^{\mathrm{L}_{1}}, w^{\prime \mathrm{L}_{1}}=\left(\frac{\lambda^{\mathrm{S}}+2 \mu^{\mathrm{S}}}{E}\right) \frac{\omega^{\prime}}{c_{1}} w^{\mathrm{L}_{1},},
\end{aligned}
$$




$$
\begin{aligned}
\tau_{z z}^{\prime \mathrm{L}_{1}} & =\frac{\tau_{z z}^{\mathrm{L}_{1}}}{E} \\
u^{\prime \mathrm{L}_{2}} & =\left(\frac{\lambda^{\mathrm{S}}+2 \mu^{\mathrm{S}}}{E}\right) \frac{\omega^{\prime}}{c_{1}} u^{\mathrm{L}_{2}}, w^{\prime \mathrm{L}_{2}}=\left(\frac{\lambda^{\mathrm{S}}+2 \mu^{\mathrm{S}}}{E}\right) \frac{\omega^{\prime}}{c_{1}} w^{\mathrm{L}_{2}} \\
\tau_{z z}^{\prime \mathrm{L}_{2}} & =\frac{\tau_{z z}^{\mathrm{L}_{2}}}{E} .
\end{aligned}
$$

In these relations $E$ is the Young's modulus of the solid phase, $\omega^{\prime}$ is a constant having the dimensions of frequency and $c_{1}$ is the velocity of a longitudinal wave propagating in a fluid saturated incompressible porous medium (Boer $e t$ al., 1993) and is given by

$$
c_{1}=\sqrt{\frac{\left(\eta^{\mathrm{F}}\right)^{2}\left(\lambda^{\mathrm{S}}+2 \mu^{\mathrm{S}}\right)}{\left(\eta^{\mathrm{F}}\right)^{2} \rho^{\mathrm{S}}+\left(\eta^{\mathrm{S}}\right)^{2} \rho^{\mathrm{F}}}} .
$$

If the pore liquid is absent or gas is filled in the pores, then $\rho^{\mathrm{F}}$ is very small as compare to $\rho^{\mathrm{S}}$ and can be neglected. So the relation (33) reduces to

$$
c_{0}=\sqrt{\frac{\lambda^{\mathrm{S}}+2 \mu^{\mathrm{S}}}{\rho^{\mathrm{S}}}} .
$$

This gives the velocity of the longitudinal wave propagating in an incompressible empty porous solid, where the change in volume is due to the change in porosity and is a wellknown result of the classical theory of elasticity. In an incompressible non-porous solid medium $\eta^{\mathrm{F}} \rightarrow 0$, then (33) becomes $c_{1}=0$ and is physically acceptable as a longitudinal wave cannot propagate in an incompressible medium.

The equations (12)-(31) with the help of (32) reduce to the dimensionless form. The differential equations for half-space are decoupled by expressing the displacement components $u^{i}$ and $w^{i}(i=\mathrm{F}, \mathrm{S})$ in terms of potential $\varphi^{i}$ and $\psi^{i}$ defined as

$$
\begin{aligned}
& u^{i}=\frac{\partial \varphi^{i}}{\partial x}+\frac{\partial \psi^{i}}{\partial z} \\
& w^{i}=\frac{\partial \varphi^{i}}{\partial z}-\frac{\partial \psi^{i}}{\partial x} .
\end{aligned}
$$

The further simplifications lead to the following set of equations for the displacements, pore pressure and stresses

$$
\begin{aligned}
& u^{\mathrm{L}_{1}}= \frac{i k}{\xi_{1}}\left\{A_{1} I_{0}^{\prime}\left(\frac{\xi_{1}(1+\alpha z)}{\alpha}\right)\right. \\
&\left.+A_{2} K_{0}^{\prime}\left(\frac{\xi_{1}(1+\alpha z)}{\alpha}\right)\right\} e^{i k(x-c t),} \\
& w^{\mathrm{L}_{1}}=\left\{A_{1} I_{0}\left(\frac{\xi_{1}(1+\alpha z)}{\alpha}\right)\right. \\
&\left.+A_{2} K_{0}\left(\frac{\xi_{1}(1+\alpha z)}{\alpha}\right)\right\} e^{i k(x-c t),} \\
& \tau_{z z}^{\mathrm{L}_{1}}=\delta^{\mathrm{L}_{1}} \frac{(1+\alpha z)}{\xi_{1}}\left(\xi_{1}^{2}-k^{2}\right)\left\{A_{1} I_{0}^{\prime}\left(\frac{\xi_{1}(1+\alpha z)}{\alpha}\right)\right. \\
&\left.+A_{2} K_{0}^{\prime}\left(\frac{\xi_{1}(1+\alpha z)}{\alpha}\right)\right\} e^{i k(x-c t)},
\end{aligned}
$$

$$
\begin{gathered}
u^{\mathrm{L}_{2}}=i k\left(A_{3} \cosh \xi_{2} z+A_{4} \sinh \xi_{2} z\right) e^{i k(x-c t)} \\
w^{\mathrm{L}_{2}}=\xi_{2}\left(A_{3} \sinh \xi_{2} z+A_{4} \cosh \xi_{2} z\right) e^{i k(x-c t)} \\
\tau_{z z}^{\mathrm{L}_{2}}=\delta^{\mathrm{L}_{2}}\left(\xi_{2}{ }^{2}-k^{2}\right)\left(A_{3} \cosh \xi_{2} z+A_{4} \sinh \xi_{2} z\right) e^{i k(x-c t)} \\
u^{\mathrm{S}}=\left(i k A_{5} e^{-\xi_{3} z}-\xi_{4} A_{6} e^{-\xi_{4} z}\right) e^{i k(x-c t)}, \\
w^{\mathrm{S}}=-\left(\xi_{3} A_{5} e^{-\xi_{3} z}+i k A_{6} e^{-\xi_{4} z}\right) e^{i k(x-c t)}, \\
\tau_{z z}^{\mathrm{S}}=\left[\left\{\xi_{3}{ }^{2}-\left(1-2 \delta^{2}\right) k^{2}\right\} A_{5} e^{-\xi_{3} z}+2 \delta^{2} A_{6} i k \xi_{4} e^{-\xi_{4} z}\right] e^{i k(x-c t)} \\
\tau_{x z}^{\mathrm{S}}=\delta^{2}\left\{-2 i k \xi_{3} A_{5} e^{-\xi_{3} z}+\left(k^{2}+\xi_{4}{ }^{2}\right) A_{6} e^{-\xi_{4} z}\right\} e^{i k(x-c t)} \\
p=-\frac{\delta_{3}{ }^{2} k c}{\left(\eta^{\mathrm{F}}\right)^{2}}\left(k c \eta^{\mathrm{S}} \delta_{4}+i P\right)\left(A_{5} e^{-\xi_{3} z}+A_{6} e^{-\xi_{4} z}\right) e^{i k(x-c t)} \\
u^{\mathrm{F}}=i\left(-\frac{\eta^{\mathrm{S}}}{\eta^{\mathrm{F}}} k A_{5} e^{-\xi_{3} z}+\gamma_{1} \xi_{4} A_{6} e^{-\xi_{4} z}\right) e^{i k(x-c t)} \\
w^{\mathrm{F}}=\left(-\frac{\eta^{\mathrm{S}}}{\eta^{\mathrm{F}}} \xi_{3} A_{5} e^{-\xi_{3} z}+\gamma_{1} k A_{6} e^{-\xi_{4} z}\right) e^{i k(x-c t)}
\end{gathered}
$$

where

$$
\begin{aligned}
& \xi_{1}^{2}=k^{2}\left(1-\delta_{1}^{2} c^{2}\right), \\
& \xi_{2}^{2}=k^{2}\left(1-\delta_{2}^{2} c^{2}\right), \\
& \xi_{3}^{2}=k^{2}\left(1-c^{2}-i \frac{Q c}{k}\right), \\
& \xi_{4}^{2}=k^{2}\left\{1-\frac{\delta_{3}^{2} c^{2}}{\delta^{2}}\left(1+i \frac{\delta_{4} P}{\left(k c \delta_{4}+i P\right)}\right)\right\}, \\
& Q=\frac{P \delta_{3}^{2}}{\left(\eta^{\mathrm{F}}\right)^{2}}, \quad \delta_{1}=\frac{c_{1}}{\alpha^{\mathrm{L}_{1}}}, \quad \alpha^{\mathrm{L}_{1}}=\sqrt{\frac{\lambda_{0}^{\mathrm{L}_{1}}}{\rho_{0}^{\mathrm{L}_{1}}}}, \\
& \delta^{\mathrm{L}_{1}}=\frac{\lambda_{0}^{\mathrm{L}_{1}}}{\lambda^{\mathrm{S}}+2 \mu^{\mathrm{S}}}, \quad \delta_{2}=\frac{c_{1}}{\alpha^{\mathrm{L}_{2}}}, \quad \alpha^{\mathrm{L}_{2}}=\sqrt{\frac{\lambda^{\mathrm{L}_{2}}}{\rho^{\mathrm{L}_{2}}}}, \\
& \delta^{\mathrm{L}_{2}}=\frac{\lambda^{\mathrm{L}_{2}}}{\lambda^{\mathrm{S}}+2 \mu^{\mathrm{S}}}, \quad \delta=\frac{\beta_{0}}{c_{0}}, \quad \beta_{0}=\sqrt{\frac{\mu^{\mathrm{S}}}{\rho^{\mathrm{S}}}}, \\
& \delta_{3}=\frac{c_{1}}{c_{0}}, \quad \delta_{4}=\frac{\rho^{\mathrm{F}}}{\rho^{\mathrm{S}}}, \quad P=\frac{S_{\mathrm{V}}}{\omega^{\prime} \rho^{\mathrm{S}}}, \\
& \gamma_{1}=\frac{P}{\delta_{4} k c+i P} .
\end{aligned}
$$

$I_{0}(z), K_{0}(z)$ are modified, zero order Bessel functions of first and second kind and primes indicate their derivatives $w . r . t$. their respective arguments. Substituting Eqs. (37)(47) in the boundary conditions (29)-(31), one can easily obtain a system of six linear simultaneous equations for the unknown arbitrary constants $A_{1}, A_{2}, A_{3}, A_{4}, A_{5}$, and $A_{6}$. The non-trivial solution of this system of equations requires that the determinant of the coefficients of the unknowns must vanish. This argument yields the required frequency equation relating the non-dimensional phase velocity $c$ with the non-dimensional wave number $k$ in the form of a sixorder determinant, which is further simplified as

$$
\tanh \xi_{2} H=\frac{a_{23} b_{32}-a_{13} R_{13}}{a_{13} b_{32}-a_{23} R_{13}},
$$


where

$$
\begin{aligned}
& a_{13}=\frac{4 \delta^{2} k^{2} \xi_{3} \xi_{4}}{R_{12}\left(k^{2}+\xi_{4}^{2}\right)}-\frac{R^{\mathrm{S}}}{R_{12}}, \\
& a_{23}=\frac{\xi_{3}}{\xi_{2}}\left(\frac{\xi_{4}^{2}-k^{2}}{\xi_{4}^{2}+k^{2}}\right), \quad a_{32}=\frac{K_{0}^{\prime}\left(\frac{\xi_{1}}{\alpha}\right)}{K_{0}\left(\frac{\xi_{1}}{\alpha}\right)} \xi_{2}, \\
& a_{35}=\frac{I_{0}^{\prime}\left(\frac{\xi_{1}}{\alpha}\right) K_{0}\left(\frac{\xi_{1}}{\alpha}\right)-I_{0}\left(\frac{\xi_{1}}{\alpha}\right) K_{0}^{\prime}\left(\frac{\xi_{1}}{\alpha}\right)}{K_{0}\left(\frac{\xi_{1}}{\alpha}\right)}, \\
& a_{52}=\frac{K_{0}^{\prime}\left(\frac{\xi_{1}(1-\alpha h)}{\alpha}\right)}{K_{0}\left(\frac{\xi_{1}}{\alpha}\right)} \xi_{2}, \quad K_{0}\left(\frac{\xi_{1}}{\alpha}\right) \\
& a_{55}=\left\{\frac{I_{0}^{\prime}\left(\frac{\xi_{1}(1-\alpha h)}{\alpha}\right) K_{0}\left(\frac{\xi_{1}}{\alpha}\right)-I_{0}\left(\frac{\xi_{1}}{\alpha}\right) K_{0}^{\prime}\left(\frac{\xi_{1}(1-\alpha h)}{\alpha}\right)}{b_{32}}=a_{32}-\frac{a_{35}}{a_{55}} a_{52}, \quad R_{13}=\frac{\lambda^{\mathrm{L}_{2}} \xi_{1}}{\lambda_{0}^{\mathrm{L}_{1}}}\left(\frac{\xi_{2}{ }^{2}-k^{2}}{\xi_{1}{ }^{2}-k^{2}}\right),\right. \\
& R_{12}=\delta^{\mathrm{L}_{2}}\left(\xi_{2}^{2}-k^{2}\right), \\
& R^{\mathrm{S}}=\xi_{3}^{2}-\left(1-2 \delta^{2}\right) k^{2}+\frac{\delta_{3}^{2}}{\left(\eta^{\mathrm{F}}\right)^{2}}\left(k^{2} c^{2} \eta^{\mathrm{S}} \delta_{4}+i P k c\right) .(55)
\end{aligned}
$$

\section{Special Cases}

Case I: If we remove the overlying inhomogeneous liquid layer by taking $h=0$, then problem reduces to the surface wave propagating in a model consist of an incompressible poro-elastic half-space lying under a uniform layer of a homogeneous liquid. The frequency equation (54) after some simplification takes the form

$$
\tanh \xi_{2} H=\frac{\xi_{2}\left\{4 \delta^{2} k^{2} \xi_{3} \xi_{4}-R^{\mathrm{S}}\left(k^{2}+\xi_{4}^{2}\right)\right\}}{\delta^{\mathrm{L}_{2}} \xi_{3}\left(\xi_{2}{ }^{2}-k^{2}\right)\left(\xi_{4}{ }^{2}-k^{2}\right)} .
$$

Case II: If we take $H=0$, then we get a fluid saturated incompressible porous half-space lying under a uniform layer of non-homogeneous layer with non-homogeneity varying with depth and the frequency equation (54) for this case reduces to

$$
a_{23} b_{32}-a_{13} R_{13}=0 .
$$

Case III: If both $h$ and $H$ are zero then Eq. (54) takes the form

$$
\begin{aligned}
4 k^{2} \xi_{3} \xi_{4} \delta^{2}= & \left(\xi_{4}^{2}+k^{2}\right)\left(\xi_{3}^{2}-\left(1-2 \delta^{2}\right) k^{2}\right. \\
& \left.+\frac{\delta_{3}^{2}}{\left(\eta^{\mathrm{F}}\right)^{2}}\left(k^{2} c^{2} \eta^{\mathrm{S}} \delta_{4}+i P k c\right)\right),
\end{aligned}
$$

which is further simplified as

$$
\begin{aligned}
& 4\left[1-\delta_{3}^{2}\left\{1+\frac{\delta_{4} P\left(P+i \delta_{4} \omega\right)}{\left(P^{2}+\delta_{4}^{2} \omega^{2}\right)}\right\}\left(\frac{c}{\delta}\right)^{2}\right]^{\frac{1}{2}} \\
& \cdot\left(1-c^{2}-i \frac{Q c}{k}\right)^{\frac{1}{2}} \\
& -\left[2-\delta_{3}^{2}\left\{1+\frac{\delta_{4} P\left(P+i \delta_{4} \omega\right)}{\left(P^{2}+\delta_{4}^{2} \omega^{2}\right)}\right\}\left(\frac{c}{\delta}\right)^{2}\right] \\
& \cdot\left\{2-\left(1-\frac{\eta^{\mathrm{S}} \delta_{4} \delta_{3}^{2}}{\left(\eta^{\mathrm{F}}\right)^{2}}\right)\left(\frac{c}{\delta}\right)^{2}\right\}=0 .
\end{aligned}
$$

This equation governs the propagation of Rayleigh type surface waves at the free surface of a fluid saturated incompressible porous half-space and if the pore liquid is absent or gas is filled in the pores then after some simplifications Eq. (59) takes the form

$$
\sqrt[4]{\left(1-\frac{c^{2}}{\delta^{2}}\right)\left(1-c^{2}\right)}=\left(2-\frac{c^{2}}{\delta^{2}}\right)^{2} .
$$

If the dimensionless quantities are converted in to the corresponding physical quantities then this equation yields

$$
\sqrt[4]{\left(1-\frac{c^{2}}{c_{0}^{2}}\right)\left(1-\frac{c^{2}}{\beta_{0}^{2}}\right)}=\left(2-\frac{c^{2}}{\beta_{0}^{2}}\right)^{2} .
$$

The above equation corresponds to the Rayleigh wave propagating in an empty incompressible porous solid and is a well-known result of classical theory.

Now we derive the equations of the trajectory of particle motion during the propagation of Rayleigh type waves in a fluid saturated incompressible porous half-space. The horizontal and vertical components of displacement of a solid particle are real valued and are provided by Eqs. (43) and (44). So after some simplifications the trajectory of a solid particle at a depth $z_{0}$ is defined by

$$
\frac{\left(u^{\mathrm{S}}\right)^{2}}{a^{2}}+\frac{\left(w^{\mathrm{S}}\right)^{2}}{b^{2}}=1 .
$$

Therefore like the classical theories, trajectory of solid particle motion in this case is also elliptic with the axes ' $a$ ' and ' $b$ ' as the real parts of the quantities $k A_{5}\left(e^{-\xi_{3} z_{0}}-\frac{2 \xi_{3} \xi_{4}}{\xi_{4}{ }^{2}+k^{2}} e^{-\xi_{4} z_{0}}\right)$ and $-\xi_{3} A_{5}\left(e^{-\xi_{3} z_{0}}-\frac{2 k^{2}}{\xi_{2}{ }^{2}+k^{2}} e^{-\xi_{4} z_{0}}\right)$ respectively and are different from their corresponding values as given in the classical theories. The vertical motion vanishes at a depth $H_{0}$ given by $H_{0}=\frac{1}{\xi_{4}-\xi_{3}} \log \left(\frac{2 k^{2}}{k^{2}+\xi_{4}^{2}}\right)$. The ellipse will then stretched out into a straight line, $u^{\mathrm{S}}=a$, and will alter its direction beyond this depth.

\section{Numerical Results and Discussions}

With a view toward illustrating the analytical procedure presented in the preceding sections, we consider an example for numerical discussion and consider a model for which the physical constants are taken from the work of Ewing et al. (1957) and Boer et al. (1993) as $\eta^{\mathrm{S}}=0.67$, $\eta^{\mathrm{F}}=0.33, \rho^{\mathrm{S}}=1.34 \mathrm{Mg} / \mathrm{m}^{3}, \rho^{\mathrm{F}}=0.33 \mathrm{Mg} / \mathrm{m}^{3}, \lambda^{\mathrm{S}}=$ $5.5833 \mathrm{MN} / \mathrm{m}^{2}, \mu^{\mathrm{S}}=8.3750 \mathrm{MN} / \mathrm{m}^{2}, k^{\mathrm{F}}=0.01 \mathrm{~m} / \mathrm{s}$, $\gamma^{\mathrm{FR}}=10.00 \mathrm{kN} / \mathrm{m}^{3}, \lambda_{0}^{\mathrm{L}_{1}}=\lambda^{\mathrm{L}_{1}}=0.214 \times 10^{10} \mathrm{~N} / \mathrm{m}^{2}$ and $\rho^{\mathrm{L}_{1}}=\rho^{\mathrm{L}_{0}^{1}}=1.0 \times 10^{3} \mathrm{~kg} / \mathrm{m}^{3}$. The results depict the variation of phase velocity with respect to wave number and their graphical representations are given in Figs. 2-6. From Figs. 2 and 3 it is evident that for any value of $\alpha h$, starting from a higher value, the phase velocity falls very quickly to some lower value then decreases gradually to the velocity of the waves of short wave length. The significant fall at the vanishing wave number is due to the damping effect of the overlying liquid layers and also the viscous damping caused by internal friction from the interaction mechanism 


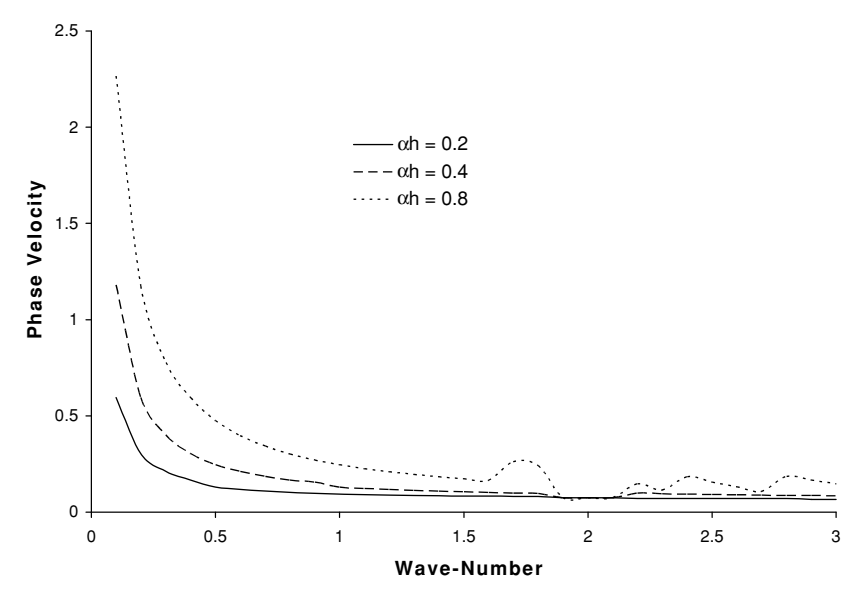

Fig. 2. Variation of phase velocity with wave-number and $\alpha h$ for $H / h=2$.

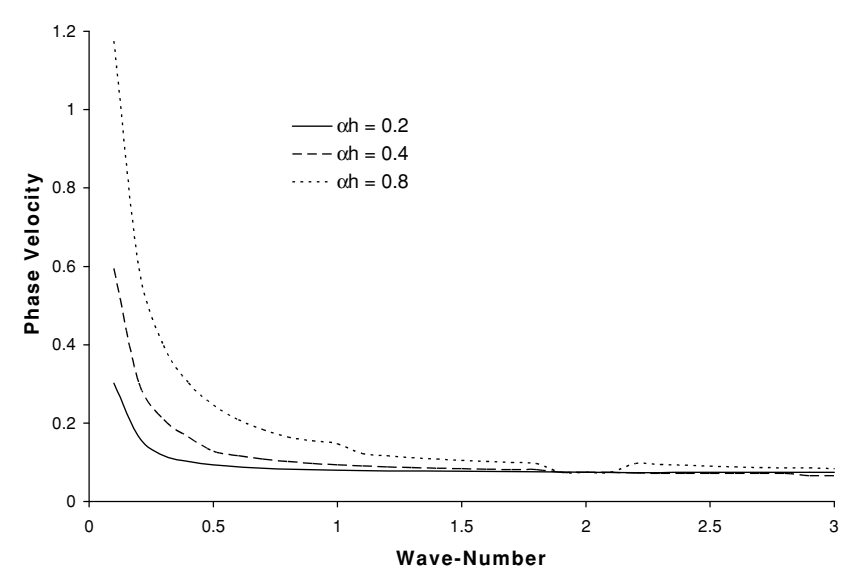

Fig. 3. Variation of phase velocity with wave-number and $\alpha h$ for $H / h=4$.

between the skeleton and pore liquid present in the pores. So in the beginning when wave number is small the waves are highly dispersive, their depressiveness decreases with increase of wave number and ultimately for all values of $\alpha h$, the phase velocity is constant and hence the waves become non-dispersive. Curves are drawn for three different values of $\alpha h$ and increase in phase velocity with increase in $\alpha h$ indicates that the non-homogeneity of the liquid layer also affects the dispersive character of the waves. Variations are shown for two values of the ratio $H / h$ and we observe that this ratio affects the phase velocity only quantitatively but not qualitatively. This is again justified when the curves are drawn for three different values of $H / h$ at some fixed values of $\alpha h$ (Figs. 4-6). It is clear that the phase velocity increases with the decrease in the values of this ratio. It is also evident that for small values of $H / h$ and large values of $\alpha h$, the dispersion curves show some kinks (like one at $k=1.7$ Fig. 2 for $H / h=2$ and $\alpha h=0.8$ ), but the decrease in non-homogeneity parameter and increase in the ratio $H / h$ make the curves smooth. Also the curves are smooth when the non-homogeneous layer is absent. So this depicts the effect of, the thickness of the homogeneous layer as compare to non-homogeneous layer and the nonhomogeneity of the upper layer, on the dispersive character

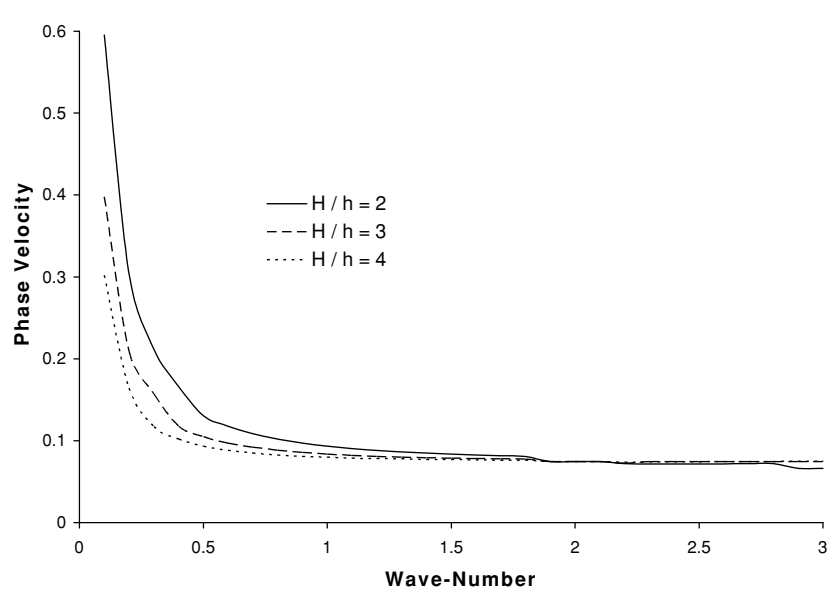

Fig. 4. Variation of phase velocity with wave-number and $H / h$ for $\alpha h=0.2$.

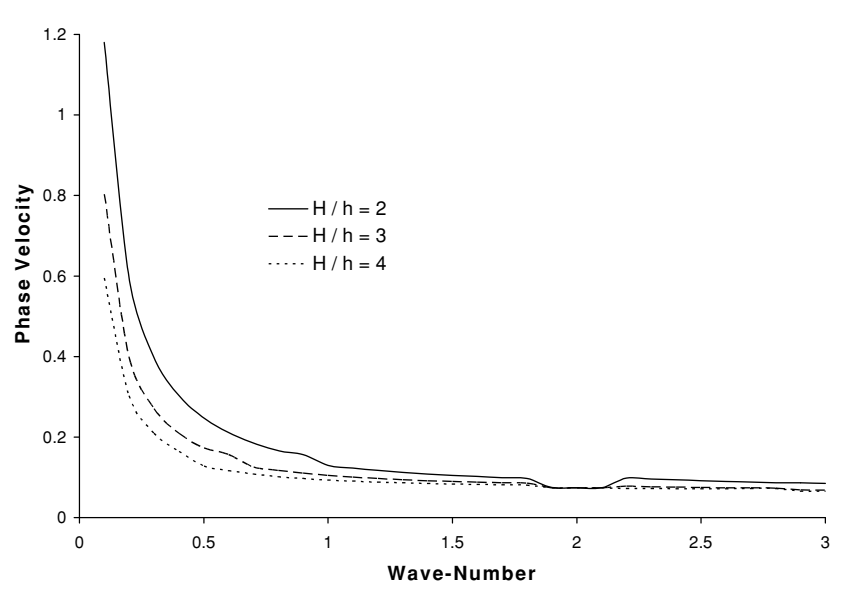

Fig. 5. Variation of phase velocity with wave-number and $H / h$ for $\alpha h=0.4$.

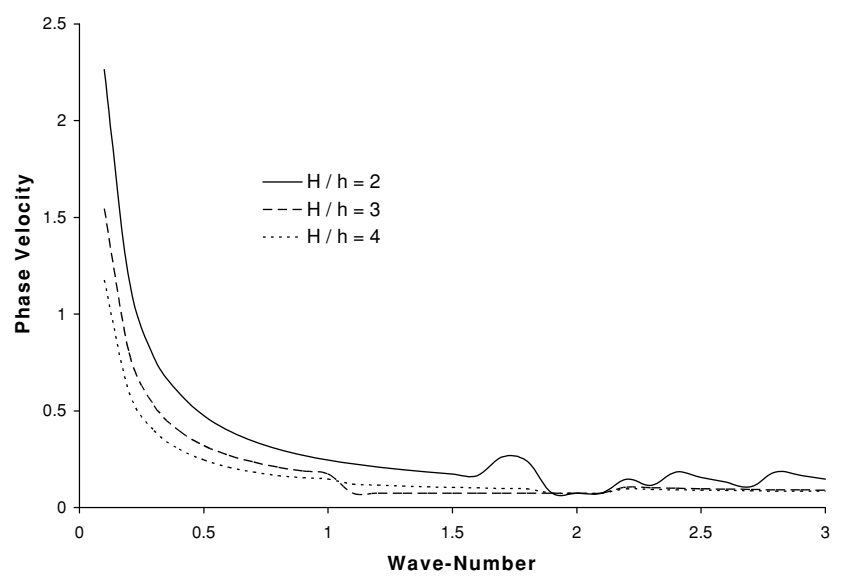

Fig. 6. Variation of phase velocity with wave-number and $H / h$ for $\alpha h=0.8$.

of the surface waves.

The variation of phase velocity with respect to wave number for the model, discussed in Case I of Section 6, is represented in Fig. 7. Here the non-homogeneous layer is absent so $h=0$. As $\tanh (i n \pi+z)=\tanh z$, so for the given values of wave number, Eq. (56) is solved for different in- 


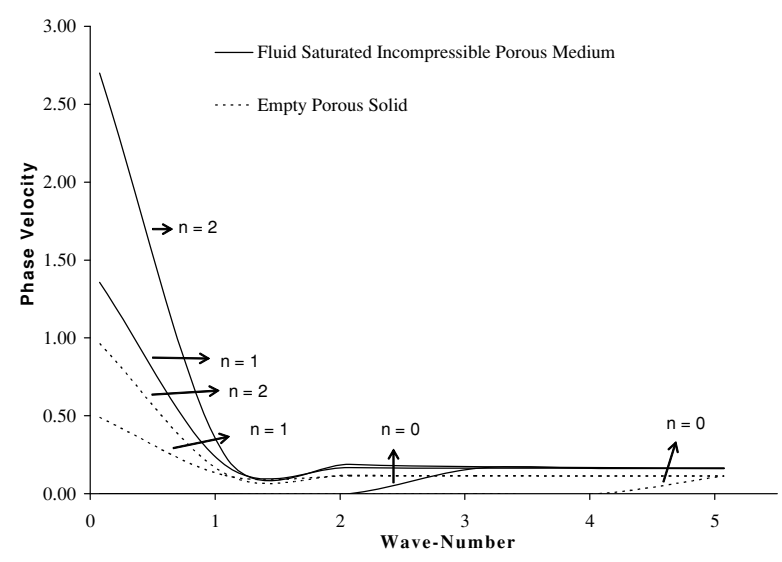

Fig. 7. Variation of phase velocity with wave-number for $h=0$.

tegral values of $n$ and for a fix value of $H$. For $n=0$, the phase velocity increases uniformly from a very small value and then becomes constant indicating that the wave for the first mode is non-dispersive. But for all other values of $n$, it is evident that the phase velocity depends upon the wave number, showing that the waves for all other modes are dispersive. Here for any value of $n$, starting from some maximum value, the phase velocity decreases very quickly to some small value, then increases and ultimately becomes constant. It again indicates that for small values of wave number, the wave is highly dispersive, but its dispersiveness decreases with the increase of wave number and ultimately it becomes non-dispersive. If the pore liquid is absent or gas is filled in the pores, then $\rho^{\mathrm{F}}$ is very small as compare to $\rho^{S}$ and hence it can be neglected. This situation for empty porous solid is represented by dotted lines in the Fig. 7 . The behavior of the dispersion curves in both the cases is almost similar, but for any value of $n$, the curves for empty porous solid lies below the corresponding curves for fluid saturated medium indicating that the presence of fluid in the pores increases the phase velocity and hence affects dispersiveness of surface waves. Another important property of the surface waves in this case is that, the phase velocity for fluid saturated as well as for empty porous medium increases with $n$ for the lower values of the wave number, but for large values of the wave number the phase velocity becomes constant and equal for all values of $n$.

\section{Conclusion}

Based on the general incompressible porous media theories, surface wave propagation in an incompressible poroelastic half-space lying under a double layer of two different liquids is discussed. The half-space is modeled as a twophase system with two incompressible constituents (porous solid and inviscid fluid), where the general field equations are directly adopted according to the work of Boer and Ehlers (1990) and Boer et al. (1993). During the analysis of the properties of the dispersion relation, it is observed that the non-homogeneity of the liquid layer, ratio of the thicknesses of the liquid layers and the presence of liquid in the pores of the incompressible porous half space have a significant effect on the dispersion of surface waves. Rayleigh type surface waves at the free surface of an incompressible porous half-space are also discussed and the results at various steps are compared with the classical theories.

\section{References}

Achenbach, J. D., Wave Propagation in Elastic Solids, North-Holland Publishing Company, Amsterdam, 1976.

Biot, M. A., General Theory of Three dimensional consolidation, J. Appl. Phys., 12, 155-161, 1941.

Biot, M. A., Theory of Propagation of Elastic Waves in a Fluid-saturated Porous Solid-I. Low Frequency Range, J. Acout. Soc. Am., 28, 168-178, 1956 a.

Biot, M. A., Theory of Propagation of Elastic Waves in a Fluid-saturated Porous Solid-II. Higher Frequency Range, J. Acout. Soc. Am., 28, 179191, $1956 b$.

Biot, M. A., Mechanics of deformation and acoustic propagation in porous media, J. Appl. Phys., 33, 1482-1498, 1962.

Bowen, R. M., Incompressible Porous Media Models by use of the Theory of Mixtures, Int. J. Engg. Sci., 18, 1129-1148, 1980.

de Boer, R., Highlights in the historical development of the porous media theory: Toward a consistent macroscopic theory, Appl. Mech. Rev., 49, 201-262, 1996.

de Boer, R., Contemporary progress in porous media theory, Appl. Mech. Rev., 53, 323-370, 2000a.

de Boer, R., Theory of porous media, Springer-Verleg, New York, 2000b. de Boer, R. and W. Ehlers, A Historical Review of the Formulation of Porous Media Theories, Acta Mechanica, 74, 1-8, 1988.

de Boer, R. and W. Ehlers, The Development of the Concept of Effective Stress, Acta Mechanica, 83, 77-92, 1990a.

de Boer, R. and W. Ehlers, Uplift, Friction and Capillarity-Three Fundamental Effects for Liquid-saturated Porous Solids, Int. J. Solid Structures, 26, 43-57, 1990b.

de Boer, R. and Z. Liu, Growth and decay of acceleration waves in incompressible saturated poroelastic solids, ZAMM, 76, 341-347, 1996.

de Boer, R., W. Ehlers, and Z. Liu, One-dimensional Transient Wave Propagation in a Fluid-saturated Incompressible Porous Media, Arch. App. Mech., 63, 59-72, 1993.

Edelman, I., Surface Waves at Vacuum/Porous Medium Interface: Low Frequency Range, Wave Motion, 39, 111-127, 2004.

Ehlers, W., Compressible, incompressible and hybrid two-phase models in porous theories, ASME; AMD, 158, 25-38, 1993.

Ewing, W. M., W. S. Jardetzky, and F. Press, Elastic Waves in Layered Media, McGraw-Hill Book Co., 1957.

Fillunger, P., Der Auftrieb in Talsperren. Osterr. Wochenschrift fur den offentl. Baudienst. I. Teil 532-552, II. Teil 552-556, III. Teil 567-570, 1933.

Kumar, R. and B. S. Hundal, A study of Spherical and Cylindrical Wave Propagation in a Non-Homogeneous Fluid Saturated Incompressible Porous Medium by Method of Characteristics, in Current Trends in Industrial and Applied Mathematics, edited by P. Manchanda et al., Anamya Publisher, New Delhi, 181-194, 2002.

Kumar, R. and B. S. Hundal, Wave Propagation in a Fluid-Saturated Incompressible Porous Medium, Indian J. Pure and Applied Math., 4, 651-665, 2003a.

Kumar, R. and B. S. Hundal, One-Dimensional Wave Propagation in a Non-Homogeneous Fluid-Saturated Incompressible Porous Medium, Bull. Allahabad Math. Soc., 18, 1-13, 2003b.

Kumar, R. and B. S. Hundal, Effect of Non-homogeneity on Onedimensional Wave Propagation in a Fluid-Saturated Incompressible Porous Medium, Bull. Cal. Math. Soc., 96, 179-188, 2004a.

Kumar, R. and B. S. Hundal, Symmetric Wave Propagation in a FluidSaturated Incompressible Porous Medium, J. Sound and Vibration, 96, 179-188, 2004b.

Kumar, R. and A. Miglani, Effect of Pore Alignment on Surface Wave Propagation in a Liquid-Saturated Porous Layer over a Liquid-Saturated Porous Half-Space with Loosely Bonded Interface, J. Phys. Earth, 44, 153-172, 1996.

Kumar, R., A. Miglani, and N. R. Garag, Surface Wave Propagation in a Double Liquid Layer over a Liquid-Saturated Porous Half-Space, Sadhana, 27, 643-655, 2002.

Liu, K. and Y. Liu, Propagation Characteristics of Rayleigh Waves in Orthotropic Fluid-Saturated Porous Media, J. Sound and Vibration, 271, 1-13, 2004.

Liu, Z., Propagation and Evolution of Wave Fronts in Two-Phase Porous Media, TIPM, 34, 209-225, 1999. 
Liu, Z. and R. de Boer, Plane waves in a semi-infinite fluid saturated porous medium, TIPM, 16, 147-173 1994.

Liu, Z. and R. de Boer, Propagation of acceleration waves in incompressible saturated porous solids, TIPM, 21, 163-173, 1995.

Liu, Z. and R. de Boer, Dispersion and Attenuation of Surface Waves in a Fluid-saturated Porous Medium, TIPM, 29, 207-223, 1997.

von Terzaghi, K., Die Berechnug der Durchlassigkeit des Tones aus dem Verlauf der hydromechanischen Spannungserscheinungen, Sitzungsber. Akad. Wiss. (Wien), Math.-Naturwiss. Kl., Abt. Iia, 132, 125-138, 1923. von Terzaghi, K., Erdbaumechanik auf Bodenphysikalischer Grundlage, 399 pp., Leipzig-wien: Franz Deuticke, 1925.

Yan, Bo, Z. Liu, and X. Zhang, Finite Element Analysis of Wave Propagation in a Fluid-saturated Porous Media, Applied Mathematics and Mechanics, 20, 1331-1341, 1999.

R. Kumar and B. S. Hundal (e-mail: hundal_bs@yahoo.co.in) 